\title{
Minimum volume standards in day surgery: a systematic review
}

\author{
Michal Stanak ${ }^{1,2^{*}}$ (D) and Christoph Strohmaier ${ }^{1}$
}

\begin{abstract}
Background: The aim was to find out if and for what indications are minimum volume standards (MVS) applied in the day surgery setting and whether the application of MVS improves patient relevant outcomes.

Methods: We conducted a comprehensive systematic literature search in seven databases on July 12th, 2019. Concerning effectiveness and safety, the data retrieved from the selected studies were systematically extracted into data-extraction tables. Two independent researchers (MS, CS) systematically assessed the quality of evidence using the quality assessment tool for individual studies of the International Society for Pharmacoeconomics and Outcomes Research (ISPOR) called Task Force Checklist for Quality Assessment of Retrospective Database Studies. No instances of disagreement occurred. No further data processing was applied.
\end{abstract}

Results: The systematic literature search, together with hand search, yielded 595 hits. No prospective or controlled studies were found. Data from eight retrospective studies were used in the analysis of clinical effectiveness and safety on seven indications: anterior cruciate ligament reconstruction, cataract surgery, meniscectomy, thyroidectomy, primary hip arthroscopy, open carpal tunnel release, and rotator cuff repair. All interventions (except for carpal tunnel release and thyroidectomy) confirmed a volume-outcome relationship (VOR) with relation to surgeon/hospital volume, however, none established MVS for the respective interventions. Safety related data were reported without its relationship to surgeon/hospital volume.

Conclusions: This present paper provides some evidence in favor of the VOR, however, it based on low quality retrospective data-analyses. The present results cannot offer any clear-cut MVS thresholds for the day surgery setting and so the simple transition from inpatient results (that support MVS) to the day surgery setting is questionable. Further quality assuring policy approaches should be considered.

\section{Background}

\section{Definition of day surgery}

The International Association for Ambulatory Surgery (IAAS) defines day surgery as a practice where patients are admitted, operated on, and discharged during the time frame of one working day (6 to $8 \mathrm{~h}$ ), with no overnight stay [1]. The EU observatory as well as the British Association for Day Surgery (BADS) further add that true day surgery includes planned non-emergency

\footnotetext{
* Correspondence: michal.stanak@protonmail.com

'Austrian Institute for Health Technology Assessment (former Ludwig Boltzmann Institute for Health Technology Assessment), Vienna, Austria ${ }^{2}$ Department of Philosophy, University of Vienna, Vienna, Austria
}

surgical procedures on carefully-selected and prepared patients that are intended to be treated in the day surgery setting $[2,3]$. Because some procedures may require longer recovery or observation, in order to keep them as day surgeries, they have to be performed in the morning sessions [3]. The US ambulatory care setting shares the definition of IAAS [4], yet at times, the US' use of the term day surgery includes $23 \mathrm{~h}$ stay surgery, which in the $\mathrm{EU}$ is seen as an inpatient surgery with a 1 day length of stay (LOS). Alternative terms conveying the same meaning in different contexts are same day surgery, ambulatory surgery, outpatient surgery, day-care hospital, day only, or zero day hospital stay. 


\section{Reasons for shifting to day surgery}

Reasons for the shift from inpatient surgery to the day surgery context are manifold: organizational, ethical, economic, and medical. The aim is to improve the pathway of care, while alleviating the distributive justice mechanism and thus saving economic resources that can be allocated elsewhere. Day surgeries allow patients to choose their own surrounding to convalesce and they are associated with shorter waiting times and lower risks of surgery cancellation [5]. Also, day surgeries claim to reduce the rates of hospital-acquired infection and venous thromboembolism [5]. Furthermore, the consequences of shifting to day surgery seem to be a win-win situation for all parties involved [5]. Omitting overnight and weekend stays can be beneficial for the patient as well as for the medical team that does not have to stay at work over those times. This, in turn, saves the resources to the payers, potentially making the surgical interventions less expensive overall. In a report published by the UK NHS in 1989, costs of day surgery were estimated to be significantly lower compared to in-patient treatment [6]. In 2015, the Kings Fund verified the initial estimates stating that increases in day surgery over the period from 1998 to 2013 have generated savings of around $£ 2$ billion $(€ 2.32$ billion) [7]. Further patient-relevant consequences of day surgery are increase of control over patients' own time and health as being called to come right before the intervention and then being followed by a telephone call after the intervention allows the patients to be more in charge [5]. Day surgery also potentially reduces the stigma that is associated with hospital admission as the patients return home within one working day.

\section{Current management in day surgery}

According to the 2019 guideline from the Association of Anesthetists (AA) and the BADS, it is thanks to the advances in surgical and anesthetic techniques that even patients with multiple comorbidities can be treated in the day care setting. Hence, AA and BADS suggest a paradigm shift towards day surgery - meaning that if inpatient surgery is being considered, it is important to question whether any strategies could be employed to enable the patient to be treated as a day case [8].

They further state that three main selection criteria need to be considered when assessing patients' eligibility: social, medical, and surgical [8]. Social criteria include the need of a responsible adult to escort the patient home and the presence of a carer for a required number of hours postoperatively in patient's home setting. Medical criteria include patients' fitness for the procedure and presence of a chronic disease. And surgical criteria require that the intervention does not carry a significant risk of serious postoperative complication that would need immediate medical attention. Postoperative symptoms must be controllable and patients should be able to mobilize before discharge. Treating a patient as a day case, hence, requires a well-organized day surgery unit that can communicate well with the patient before, during, as well as after the intervention. It is needed because patients should be admitted to the day surgery unit as close as possible to the time of their surgery and followed-up after the surgery once in their own home setting [8]. Patients should be provided with general, as well as procedure-specific, information that should be given to them in advance of admission and verbal comments should be reinforced with written material [8].

In terms of premises, day surgeries can be conducted in hospital outpatient departments, freestanding ambulatory surgery centers, or in office-based surgeries [4]. Also, the same hospital beds used for inpatient surgeries can be used for day surgery procedures. Concerning equipment and supplies, the same is needed for conducting inpatient as well as day surgery interventions, except for the presence of overnight beds.

\section{Theory of minimum volume standards}

The theory of minimum volume standards (MVS) is based on the assumption that practice makes perfect. The assumption is that there is a relationship between volume and outcome, meaning that physicians, nonphysician staff, or hospitals improve their (surgical) capabilities and outcomes with increasing volume of patients through a learning effect. The expected causality is that quantity affects quality. On the basis of available inpatient data on the volume-outcome relationship (VOR), health care decision-makers promote the implementation of regulatory MVS frameworks that should assure that surgeons or hospitals that comply with MVSs deliver their services at a certain level of quality. In most cases, annual volumes per surgeon or hospital are applied. A possible consequence of not complying with MVS is that surgeries are not reimbursed anymore [9].

In the absence of systematic reviews on MVS in the day surgery setting, we were commissioned by the Main Association of Austrian Social Security Institutions to conduct a systematic review on the topic. Our aim was to find out if and for what indications are MVS applied in the day surgery setting and whether the application of MVS improves patient relevant outcomes. For our review, we used the European Network for Health Technology Assessment (EUnetHTA) Core Model $^{\circ}$ for Rapid Relative Effectiveness Assessment (REA) and we adjusted the assessment elements to give us answers on the impact of MVS on clinical outcomes. 


\section{Methods}

\section{Systematic literature search and study selection}

We conducted a comprehensive systematic literature search on July 12, 2019 in MEDLINE via OVID, Embase, the Cochrane Library, CRD (DARE, NHSEED, HTA), and Livivo. The search was limited to years 2000 to 2019 and to articles published in English or German. The inclusion criteria for literature selection were defined using Population-InterventionComparison-Outcome-(Study design) model shown in Table 1. While no minimum number on patient population was applied, individual case reports were excluded. We did not search for specific interventions, but for any interventions that provided information on the VOR, or more specifically on the MVS. The target patient population was hence identified by the interventions with VOR/MVS.

\section{Analysis and synthesis}

For the systematic literature search on effectiveness and safety, the data retrieved from the selected studies were systematically extracted into data-extraction tables (see Tables 2 and 3). No further data processing such as indirect comparison was applied. Two independent researchers (MS, CS) systematically assessed the quality of evidence using the quality assessment tool for individual studies of the International Society for Pharmacoeconomics and Outcomes Research (ISPOR) called Task Force Checklist for Quality Assessment of Retrospective Database Studies [18]. No instances of disagreement occurred. Due to the retrospective study design of the evidence base, data on each selected outcome category were not synthesized across studies according to
Grading of Recommendations Assessment, Development and Evaluation (GRADE).

Outcome measures were specified once interventions with VOR/MVS were found. Crucial outcomes were selected according to EUnetHTA guidelines [19, 20] that suggest using clinical outcomes relevant for patients (mortality, morbidity, health-related quality of life, and satisfaction) and not surrogate end points. The eight studies included reported on seven different indications: primary hip arthroscopy, carpal tunnel release, rotator cuff repair, ACL reconstruction, meniscectomy, thyroidectomy, and cataract surgery. The description of intervention and the outcomes assessed in relation to volume (surgical/hospital) are listed below in Table 4 .

\section{Results}

Overall, 538 citations were included after deduplication and the specific search strategies can be found in the supplementary material. Additional 57 citations we found via hand search, which resulted in overall 595 hits. No prospective or controlled studies were found by the systematic literature search and one of the studies had a control group [21]. Data from eight retrospective studies (and seven indications) were used in the analysis of clinical effectiveness and safety. Two studies were found on the intervention of anterior cruciate ligament (ACL) reconstruction [22, 23], two on cataract surgery $[24,25]$, and one for each of the following interventions: meniscectomy, thyroidectomy, primary hip arthroscopy, open carpal tunnel release, and rotator cuff repair [21, 26-28].

Table 1 PICO inclusion criteria

\begin{tabular}{|c|c|}
\hline Population & $\begin{array}{l}\text { Patients suitable for day surgery, for example: } \\
\beta \text { according to anesthesia risk classes (ÖGARI), } \\
\beta \text { according to the type of anesthetic options (local, mask), } \\
\beta \text { according to ASA classification or general illness/condition. } \\
\text { The appropriate patients are identified in the literature analysis } \\
\text { for the specific interventions/indications found. } \\
\text { Key words: day surgery, same day surgery, ambulatory surgery, outpatient surgery, day-care hospital, day only, zero-day hospital } \\
\text { stays }\end{array}$ \\
\hline Intervention & $\begin{array}{l}\text { Identified surgical interventions from the international literature in the day surgery setting that implement minimum volume } \\
\text { standards. }\end{array}$ \\
\hline Control & The same or comparable surgical interventions in the day surgery setting without minimum volume standards implemented. \\
\hline Outcomes & $\begin{array}{l}\text { Depending on the identified indications/interventions, general health-relevant results such as morbidity, mortality, functional out- } \\
\text { comes such as functionality in everyday life or at the workplace, quality of life or satisfaction are taken into account. In addition, re- } \\
\text { sults are specifically considered for outpatient interventions such as frequency of hospital infections and venous thrombosis } \\
\text { embolisms, etc. }\end{array}$ \\
\hline Setting & Day surgery/outpatient care/day clinic/zero-day stays/same-day surgery \\
\hline Study design & No limitation \\
\hline $\begin{array}{l}\text { Publication } \\
\text { period }\end{array}$ & 2000-2019 \\
\hline Language & English/German \\
\hline
\end{tabular}


Table 2 from retrospective database analyses (joints and carpal tunnel)

\begin{tabular}{|c|c|c|c|c|c|}
\hline & Degen et al. [10] (2017) & Evers et al. [11] (2018) & Jain et al. [12] (2005) & Jain et al. [13] (2004) & $\begin{array}{l}\text { Liu et al. [14] } \\
\text { (2018) }\end{array}$ \\
\hline Country & USA & The Netherlands & USA & USA & USA \\
\hline Sponsor & $\begin{array}{l}\text { National Institute of } \\
\text { Health/ National Institute } \\
\text { for Arthritis and } \\
\text { Musculoskeletal and Skin } \\
\text { Diseases (R01AR066069) }\end{array}$ & $\begin{array}{l}\text { National Institute of } \\
\text { Health/ National Institute } \\
\text { of Arthritis and } \\
\text { Musculoskeletal and Skin } \\
\text { Diseases (RO1 AR82813) }\end{array}$ & NA & NA & NA \\
\hline $\mathrm{COI}$ & $\begin{array}{l}1 \text { author (B.T.K) has COI } \\
\text { due to fees form Arthrex } \\
\text { and } \\
\text { A-3 surgical }\end{array}$ & None & NA & NA & None \\
\hline Study design & $\begin{array}{l}\text { Retrospective database } \\
\text { analysis of } 137 \text { surgical } \\
\text { centres (multivariate } \\
\text { regression) }\end{array}$ & $\begin{array}{l}\text { Retrospective database } \\
\text { analysis of } 11 \text { surgical } \\
\text { centres (univariate and } \\
\text { multivariate regression) }\end{array}$ & $\begin{array}{l}\text { Retrospective database } \\
\text { analysis of unclear } \\
\text { number of surgical } \\
\text { centres (univariate and } \\
\text { multivariate regression) }\end{array}$ & $\begin{array}{l}\text { Retrospective database } \\
\text { analysis of unclear } \\
\text { number of surgical } \\
\text { centres (multivariate } \\
\text { logistic and linear } \\
\text { regression) }\end{array}$ & $\begin{array}{l}\text { Retrospective } \\
\text { database analysis of } \\
\text { unclear number of } \\
\text { surgical centres } \\
\text { (multivariate } \\
\text { regression) }\end{array}$ \\
\hline Conducted in & 1998-2012 & $2011-2015$ & 1997-2000 & $1997-2000$ & 2009-2013 \\
\hline Indication & Hip arthroscopy & Carpal tunnel syndrome & $\begin{array}{l}\text { See inclusion criteria } \\
\text { below }\end{array}$ & $\begin{array}{l}\text { See inclusion criteria } \\
\text { below }\end{array}$ & $\begin{array}{l}\text { Anterior cruciate } \\
\text { ligament injury }\end{array}$ \\
\hline Intervention & Primary hip arthroscopy & $\begin{array}{l}\text { Open carpal tunnel } \\
\text { releases }\end{array}$ & Rotator cuff repair & $\begin{array}{l}\mathrm{ACL} \text { reconstruction \& } \\
\text { Meniscectomy }\end{array}$ & $\mathrm{ACL}$ reconstruction \\
\hline Setting & Outpatient & Outpatient & Outpatient & Outpatient & Outpatient \\
\hline $\begin{array}{l}\text { Type of } \\
\text { volume } \\
\text { analysis } \\
\text { (surgeon/ } \\
\text { hospital) }\end{array}$ & Surgeon & Surgeon & Surgeon \& hospital & Surgeon \& hospital & Hospital \\
\hline Comparator & NA & NA & NA & NA & NA \\
\hline $\begin{array}{l}\text { Number of } \\
\text { pts }\end{array}$ & 7836 & $2057^{\mathrm{a}}$ & $10,934^{b}$ & $\begin{array}{l}\text { ACL: } 32,440 \\
\text { Men: } 195,597\end{array}$ & 26,873 \\
\hline $\begin{array}{l}\text { Number of } \\
\text { procedures }\end{array}$ & $8267^{d}$ & 2057 & NA & NA & NA \\
\hline $\begin{array}{l}\text { Number of } \\
\text { surgeons }\end{array}$ & 295 & 17 & NA & NA & NA \\
\hline $\begin{array}{l}\text { Number of } \\
\text { hospitals }\end{array}$ & 137 & 11 & NA & NA & NA \\
\hline $\begin{array}{l}\text { Surgeon volum } \\
\text { year) }\end{array}$ & e categories, $n$ (cases/ & & & ACL/Men: & \\
\hline$\beta$ Low & $<102$ & $6-44$ & $<15^{\mathrm{e}}$ & $<25^{\mathrm{f}} /<75$ & NA \\
\hline$\beta$ Medium & $102 \leq x<164$ & $47-71$ & $15 \leq x<30$ & $25 \leq x<75 / 75 \leq x<175$ & \\
\hline$\beta$ High & $164 \leq x<340$ & $75-163$ & $\geq 30$ & $\geq 75 / \geq 175$ & \\
\hline$\beta$ Very high & $\geq 340$ & NA & NA & NA & \\
\hline \multicolumn{3}{|c|}{$\begin{array}{l}\text { Hospital volume categories, } n \text { (cases/ } \\
\text { year) }\end{array}$} & & ACL/Men: & \\
\hline$\beta$ Low & NA & NA & $<75$ & $<125 /<600^{9}$ & $<1006$ \\
\hline$\beta$ Medium & NA & NA & $\geq 75-<200$ & $\begin{array}{l}125 \leq x<300 / 600 \leq x< \\
1200\end{array}$ & $\geq 100-<500$ \\
\hline$\beta$ High & NA & NA & $\geq 200$ & $\geq 300 / \geq 1200$ & $\geq 500$ \\
\hline $\begin{array}{l}\text { Operating } \\
\text { time, median } \\
\text { in min } \\
\text { (range) }\end{array}$ & NA & NA & $102(30-595)^{\mathrm{h}}$ & $\begin{array}{l}\text { ACL: } 125(\mathrm{NA}) \\
\text { Men: } 55(\mathrm{NA})\end{array}$ & NA \\
\hline $\begin{array}{l}\text { Inclusion } \\
\text { criteria }\end{array}$ & $\begin{array}{l}\text { HA for diagnosis with or } \\
\text { without synoval biopsy, }\end{array}$ & $\begin{array}{l}\text { Consent, primary carpal } \\
\text { tunnel release, baseline }\end{array}$ & $\begin{array}{l}\text { Rupture of the rotator } \\
\text { cuff, disorders of bursae }\end{array}$ & $\begin{array}{l}\text { ACL: Complete rupture of } \\
\text { old disruption of } A C L \text { and }\end{array}$ & NA \\
\hline
\end{tabular}


Table 2 from retrospective database analyses (joints and carpal tunnel) (Continued)

\begin{tabular}{|c|c|c|c|c|c|}
\hline & Degen et al. [10] (2017) & Evers et al. [11] (2018) & Jain et al. [12] (2005) & Jain et al. [13] (2004) & $\begin{array}{l}\text { Liu et al. [14] } \\
\text { (2018) }\end{array}$ \\
\hline & $\begin{array}{l}\text { HA for removal of loose/ } \\
\text { foreign body, HR \& } \\
\text { chondroplasty, abrasion } \\
\text { arthroplasty \& resection } \\
\text { of labrum, HA \& } \\
\text { synovectomy, HA with } \\
\text { femoroplasty, HA with } \\
\text { acetabuloplasty, HA with } \\
\text { labral repair, total hip } \\
\text { replacement, resurfacing } \\
\text { hip \& partial/total } \\
\text { acetabulum \& femoral } \\
\text { head }\end{array}$ & $\begin{array}{l}\text { and follow-up measure- } \\
\text { ment of BCTQ }\end{array}$ & $\begin{array}{l}\text { and tendon, sprains and } \\
\text { strains of the rotator cuff } \\
\text { capsule }\end{array}$ & $\begin{array}{l}\text { sprain of cruciate } \\
\text { ligament of the knee } \\
\text { Men: derangement, } \\
\text { bucket handle tear, } \\
\text { simple tear of the } \\
\text { meniscus or cartilage }\end{array}$ & \\
\hline $\begin{array}{l}\text { Exclusion } \\
\text { criteria }\end{array}$ & NA & $\begin{array}{l}\text { Unavailable operative } \\
\text { report, unidentified } \\
\text { surgeon, cases in which } \\
\text { surgeons did not perform } \\
\text { CTRs for at least } 1 \text { year } \\
\text { within the cohort }\end{array}$ & $\begin{array}{l}\text { Shoulder bone infection, } \\
\text { present surgery as } \\
\text { corrective surgery, } \\
\text { malignancy, pathologic } \\
\text { fracture, fracture due to } \\
\text { injury in the bones of the } \\
\text { shoulder region, or } \\
\text { simultaneous total or } \\
\text { partial shoulder } \\
\text { arthroplasty }\end{array}$ & $\begin{array}{l}\text { ACL/Men: Lower leg bone } \\
\text { infections like } \\
\text { osteomyelitis, } \\
\text { inflammatory reaction } \\
\text { due to graft, correction } \\
\text { surgery, malignancies or } \\
\text { pathological fractures, } \\
\text { fractures due to injury, } \\
\text { simultaneous knee } \\
\text { arthroplasty, rheumatoid } \\
\text { arthritis, operating } \\
\text { time }<45 \text { min \& }<20 \text { min } \\
\text { in case of meniscectomy }\end{array}$ & $\begin{array}{l}\text { Non-New York } \\
\text { residents, pts. that } \\
\text { left against medical } \\
\text { advice, mortalities, } \\
\text { pts. < } 18 \text { yrs., } \\
\text { surgeries from } \\
\text { December } 2013\end{array}$ \\
\hline $\begin{array}{l}\text { Co- } \\
\text { interventions }\end{array}$ & NA & $\begin{array}{l}\text { Trigger finger release, } \\
\text { cubital tunnel relese, } \\
\text { Guyon release, radial } \\
\text { tunnel release, fasciotomy } \\
\text { Dupuytren, other, } \\
\text { standard postoperative } \\
\text { care - nerve and tendon- } \\
\text { gliding exercises }\end{array}$ & NA & NA & NA \\
\hline $\begin{array}{l}\text { Age, mean, } \\
\text { yrs (range) }\end{array}$ & $38(7-84)$ & $54(41-67)$ & $56(43.6-68.4)$ & $\begin{array}{l}\text { ACL: } 29.4(18.9-39.9) \\
\text { Men: } 47.3(31.9-62.7)\end{array}$ & $\begin{array}{l}\text { Average } 33.3(22.0- \\
46.6)\end{array}$ \\
\hline $\begin{array}{l}\text { Sex, female: } \\
\text { male, } n\end{array}$ & 4443:3801 & 986:359 & $3785-6188$ & $\begin{array}{l}\text { ACL: } 7481: 10,908^{\mathrm{i}} \\
\text { Men: } 50,108: 72,889^{\mathrm{j}}\end{array}$ & $9811: 17,049$ \\
\hline $\mathrm{BMI} \pm \mathrm{SD}$ & NA & $27 \pm 5$ & NA & NA & NA \\
\hline Comorbidities & NA & $\begin{array}{l}\text { Diabetes mellitus, } \\
\text { Rheumatoid arthritis, } \\
\text { Dupuytren's disease, } \\
\text { Trigger fingers, CMCI joint } \\
\text { arthritis, compression } \\
\text { neuropathy, tendinitis, } \\
\text { history of wrist trauma, } \\
\text { scaphotrapeziotrapezoidal } \\
\text { joint arthritis, radiocarpal } \\
\text { arthritis, peripheral } \\
\text { neuropathy, cervical } \\
\text { radioculopathy, ulnocarpal } \\
\text { impingement }\end{array}$ & Mean Deyo score: 0.1-0.9 & NA & $\begin{array}{l}\text { Mean Deyo score: } \\
0.06\end{array}$ \\
\hline $\begin{array}{l}\text { Follow-up } \\
\text { time, yrs }\end{array}$ & $10^{k}$ & 0.5 & NA & NA & 30 days \\
\hline $\begin{array}{l}\text { Patients } \\
\text { excluded } \\
\text { from the } \\
\text { analysis, } n \\
\text { (\%) }\end{array}$ & $?$ & 712 & 961 & $\begin{array}{l}\text { ACL: } 14,050 \\
\text { Men: } 72,585\end{array}$ & NA \\
\hline \multicolumn{6}{|l|}{ Efficacy } \\
\hline Survival, \% & At $2 / 5 / 10 \mathrm{yrs}$ & & & & \\
\hline
\end{tabular}


Table 2 from retrospective database analyses (joints and carpal tunnel) (Continued)

\begin{tabular}{llllll}
\hline & Degen et al. [10] (2017) & Evers et al. [11] (2018) & Jain et al. [12] (2005) & Jain et al. [13] (2004) & $\begin{array}{l}\text { Liu et al. [14] } \\
\text { (2018) }\end{array}$ \\
\hline B Low & $86.5 / 78.5 / 72.7$ & NA & NA & NA & NA \\
B Medium & $87.8 / 82.7 / 82.7$ & & & \\
$\beta$ H High & $94.6 / 90.2 / 90.2$ & & & \\
$\beta$ Very high & $97.6 / 97.6 / 97.6$ & & &
\end{tabular}

Reduction in pain score units on VAS scale, baseline/6 mo by volume, mean $\mathbf{n}$ (SD)

\begin{tabular}{|c|c|c|c|c|c|}
\hline$\beta$ Low & NA & Low: 47/18' & NA & NA & NA \\
\hline$\beta$ Medium & & Medium: 51/20 & & & \\
\hline$\beta$ High & & High: 51/19 & & & \\
\hline$\beta P$ value & & NA & & & \\
\hline \multicolumn{6}{|l|}{ ЗCTQ, mean n } \\
\hline $\begin{array}{l}\beta \text { Symptom } \\
\text { severity } \\
\text { score }\end{array}$ & NA & $\begin{array}{l}\text { Low\&Medium\&High: all } \\
1.7^{\mathrm{m}}\end{array}$ & NA & NA & NA \\
\hline $\begin{array}{l}\beta \text { Functional } \\
\text { status score }\end{array}$ & NA & $\begin{array}{l}\text { Low\&Medium\&High: all } \\
1.7\end{array}$ & NA & NA & NA \\
\hline
\end{tabular}

Hospital based acute care within 30 days, odds ratio $n$ at 5 years ( $p$ value)

\begin{tabular}{|c|c|c|c|c|c|}
\hline $\begin{array}{l}\beta \text { Low } \\
\text { volume }\end{array}$ & NA & NA & NA & NA & $x$ \\
\hline $\begin{array}{l}\beta \text { Medium } \\
\text { volume }\end{array}$ & NA & NA & NA & NA & 0.77 ( $p$ 0.059) \\
\hline $\begin{array}{l}\beta \text { High } \\
\text { volume }\end{array}$ & NA & NA & NA & NA & $0.47(p<0.001)$ \\
\hline
\end{tabular}

Risk of reoperation, hazard ratio $\mathrm{n}(95 \% \mathrm{Cl})$

\begin{tabular}{|c|c|}
\hline $\begin{array}{l}\beta \text { Low } \\
\text { volume }\end{array}$ & $x$ \\
\hline $\begin{array}{l}\beta \text { Medium } \\
\text { volume }\end{array}$ & $0.9 x(\mathrm{Cl}-0.74)$ \\
\hline $\begin{array}{l}\beta \text { High } \\
\text { volume }\end{array}$ & 0.42 (Cl 0.32) \\
\hline $\begin{array}{l}\beta \text { Very high } \\
\text { volume }\end{array}$ & $0.17(\mathrm{Cl} 0.07)$ \\
\hline
\end{tabular}

Nonroutine disposition of pts at discharge ${ }^{n}$, surgeon, $\mathrm{n}(95 \% \mathrm{Cl})$

\begin{tabular}{|c|c|c|c|c|}
\hline $\begin{array}{l}\beta \text { Low } \\
\text { volume }\end{array}$ & NA & NA & $2.8 \times(0.9-9.1)$ & ACL: $0.9 \%$ Men: $1.4 \%$ \\
\hline $\begin{array}{l}\beta \text { Medium } \\
\text { volume }\end{array}$ & NA & NA & $1.5 \times(0.7-3.1)$ & ACL: $0.4 \%$ Men: $0.7 \%$ \\
\hline $\begin{array}{l}\beta \text { High } \\
\text { volume }\end{array}$ & NA & NA & $x$ & ACL: $0.2 \%$ Men: $0.5 \%$ \\
\hline
\end{tabular}

Nonroutine disposition of pts at discharge, hospital, $\mathrm{n}(95 \% \mathrm{Cl})$

\begin{tabular}{|c|c|c|c|c|}
\hline $\begin{array}{l}\beta \text { Low } \\
\text { volume }\end{array}$ & NA & NA & $2.1 \times(0.6-8.0)$ & ACL: 1\% Men: 1.6\% \\
\hline $\begin{array}{l}\beta \text { Medium } \\
\text { volume }\end{array}$ & NA & NA & $1.7 x(0.2-11.6)$ & ACL: $0.2 \%$ Men: $1.2 \%$ \\
\hline $\begin{array}{l}\beta \text { High } \\
\text { volume }\end{array}$ & NA & NA & $x$ & ACL: $0.3 \%$ Men: $0.2 \%$ \\
\hline
\end{tabular}

Mean operating time volume, surgeon, $\min ( \pm S D)$

\begin{tabular}{|c|c|c|c|c|}
\hline $\begin{array}{l}\beta \text { Low } \\
\text { volume }\end{array}$ & NA & NA & $112(4)$ & ACL: 149(9) Men: 72(6) \\
\hline $\begin{array}{l}\beta \text { Medium } \\
\text { volume }\end{array}$ & NA & NA & $113(4)$ & ACL: 137(9) Men: 64(6) \\
\hline
\end{tabular}


Table 2 from retrospective database analyses (joints and carpal tunnel) (Continued)

\begin{tabular}{|c|c|c|c|c|c|}
\hline & Degen et al. [10] (2017) & Evers et al. [11] (2018) & Jain et al. [12] (2005) & Jain et al. [13] (2004) & $\begin{array}{l}\text { Liu et al. [14] } \\
\text { (2018) }\end{array}$ \\
\hline $\begin{array}{l}\beta \text { High } \\
\text { volume }\end{array}$ & NA & NA & $102(4)$ & ACL: 122(9) Men: 53(6) & NA \\
\hline$\beta p$ value & NA & NA & $<0.001$ & NA & NA \\
\hline \multicolumn{6}{|c|}{ Mean operating time volume, hospital, $\min ( \pm S D)$} \\
\hline $\begin{array}{l}\beta \text { Low } \\
\text { volume }\end{array}$ & NA & NA & 111 & ACL: 150(9) Men: 71(5)15 & NA \\
\hline $\begin{array}{l}\beta \text { Medium } \\
\text { volume }\end{array}$ & NA & NA & 109 & ACL: 132(9) Men: 66(6) & NA \\
\hline $\begin{array}{l}\beta \text { High } \\
\text { volume }\end{array}$ & NA & NA & 105 & ACL: 129(14) Men: 52(6) & NA \\
\hline \multicolumn{6}{|c|}{ LOS and surgeon volume, $\mathrm{n}(95 \% \mathrm{Cl})$} \\
\hline $\begin{array}{l}\beta \text { Low } \\
\text { volume }\end{array}$ & NA & NA & $2.3 \times(1.2-4.4)$ & NA & NA \\
\hline $\begin{array}{l}\beta \text { Medium } \\
\text { volume }\end{array}$ & NA & NA & $1.3 \times(0.7-2.6)$ & NA & NA \\
\hline $\begin{array}{l}\beta \text { High } \\
\text { volume }\end{array}$ & NA & NA & $x$ & NA & NA \\
\hline \multicolumn{6}{|c|}{ LOS and hospital volume, $\mathrm{n}(95 \% \mathrm{Cl})$} \\
\hline $\begin{array}{l}\beta \text { Low } \\
\text { volume }\end{array}$ & NA & NA & $0.5 \times(0.2-1.1)$ & NA & NA \\
\hline $\begin{array}{l}\beta \text { Medium } \\
\text { volume }\end{array}$ & NA & NA & $1.1 \times(0.4-3.1)$ & NA & NA \\
\hline $\begin{array}{l}\beta \text { High } \\
\text { volume }\end{array}$ & NA & NA & $x$ & NA & NA \\
\hline \multicolumn{6}{|l|}{ Safety } \\
\hline SAE & NA & NA & NA & NA & NA \\
\hline AEs, $n(\%)$ & $\mathrm{NA}(0.2)^{\mathrm{p}}$ & $23(1.6)$ & NA & NA & Unclear ${ }^{q}$ \\
\hline $\begin{array}{l}\beta \text { Day surgery } \\
\text { related AEs }\end{array}$ & $\begin{array}{l}\text { MI, ileus, pneumonia, } \\
\text { sepsis, mechanical } \\
\text { complication, hardware } \\
\text { failure, DVT/PE, wound } \\
\text { infection, dislocation/ } \\
\text { iatrogenic instability, } \\
\text { major bleed }^{r}\end{array}$ & $\begin{array}{l}\text { Wound infection, wound } \\
\text { dehiscence }\end{array}$ & NA & NA & NA \\
\hline
\end{tabular}

$A C L$ Anterior cruciate ligament, BCTQ Boston Carpal Tunnel Questionnaire, COI Conflict of interests, CTR Carpal tunnel release, DVT Deep vein thrombosis, ED Emergency department, HA Hip arthroscopy, LOS Length of stay, THA Total hip arthroplasty, Men Meniscectomy, MI Myocardial infarction, NA Not available, PE Pulmonary embolism

${ }^{\mathrm{a}} 1345$ pts. included in the analysis

${ }^{\mathrm{b}}$ Number of pts. finally included in the analysis was 9973 . Exclusion criteria were applied to exclude diagnoses, which outcomes were expected to differ from outcomes of the included indications

cNumber of pts. finally included in the analysis was 18,390 for $\mathrm{ACL}$ and 123,012 for meniscectomy

${ }^{d}$ Including 23 simultaneous bilateral and 408 staged bilateral procedures

${ }^{\mathrm{e}}$ Number of cases in the period of $1997-2000$

f Number of cases per 4 years

${ }^{9}$ Number of cases per 5 years

hata missing on 123 pts. $(0.01 \%)$

iRecords missing on $1 \mathrm{pt}$

${ }^{\mathrm{j}}$ Records missing on 15 pts

${ }^{\mathrm{k}}$ Analysis of volume/risk of reoperation relationship assessed at 5 years

'The scores were reported only in a table and so the following numbers are only authors' estimates

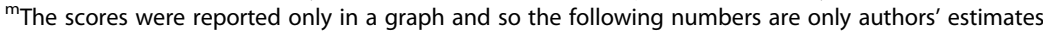

${ }^{n}$ Nonroutine disposition included transfer to another hospital, skilled nursing facility, intermediate care facility, or home health care. Routine disposition reflected patients who were discharged home

${ }^{\circ}$ Only restricted to the New York state database pts

PThirty days procedural complication rate

${ }^{\mathrm{q}}$ Listed complication are not necessarily intervention related, they are merely the reasons due to which pts. visited EDs within 30 days of ACL surgery

${ }^{r}$ Not reported in what $\mathrm{n}$ of pts., nor in relationship to surgeon volume 
Table 3 Results from retrospective database analyses (thyroid and cataract surgery)

\begin{tabular}{|c|c|c|c|}
\hline & Ayala and Yencha [15] (2015) & Chen et al. [16] (2014) & Keay et al. [17] (2012) \\
\hline Country & USA & USA & USA \\
\hline Sponsor & NA & NA & $\begin{array}{l}\text { National Eye Institute: R01EY016769. K.K } \\
\text { funded by an Australian National Health and } \\
\text { Medical Research Council post-doctoral fellow } \\
\text { ship. E.W.G. recipient of an Ernest and Eliza- } \\
\text { beth Althouse Special Scholar's Award from } \\
\text { Research to Prevent Blindness. }\end{array}$ \\
\hline
\end{tabular}

\section{Conflict of} Interest

Study design

\section{Conducted in}

Indication

Intervention

One author (Yencha) was involved in all cases either as primary or assistant surgeon

Retrospective single centre analysis

2006-2014

\section{Setting}

Type of volume

analysis

(surgeon/

hospital)

Comparator

Inpatient Thyroid surgery/Thyroidectomy

Number of pts, I $160(109$ vs 51)

vs $\mathrm{C}$

Number of

Total: 35 vs 26

procedures, I vs

$\mathrm{C}$

Benign or malignant thyroid carcinoma

Outpatient thyroid surgery/Thyroidectomy

Outpatient and Inpatient ${ }^{a}$

Number of

surgeons

Number of

hospitals

Surgeon

Surgeon volume

categories, $\mathbf{n}$

(cases/year)

\section{Hospital volume Unclear \\ categories, $\mathbf{n}$ \\ (cases/year)

\author{
Operating time, NA \\ median in min \\ (range) \\ Inclusion criteria Patients in ASA class \\ $1,2,3$ and 4
}

Exclusion criteria NA

ASA class, $\mathbf{n}, \mathbf{I}$ vs 1 and 2: 90 vs 39

$$
\text { C } 3 \text { and 4: } 19 \text { vs } 12
$$

Co-interventions Intravenous dexamethasone, intravenous antibiotics, anaesthesia at surgeon's discretion, Prophylactic calcium carbonate and vitamin D

None

Retrospective single centre chart review

2011-2012

Cataract

Cataract Surgery

(Phacoemulsification)

Outpatient Surgical Centre

Surgeon

None

Retrospective analysis of Medicare beneficiary claims data

2003-2004

Cataract

Cataract Surgery

Outpatient surgery centres

Surgeon

NA

3339

NA

4

1

Surgeon 4: 411

Surgeon 1: 536

Surgeon 2: 1056

Surgeon 3: 1336

NA

NA

Use of topical anaesthesia and performance of the intervention at an outpatient centre/setting

Patients requiring additional anaesthesia and those who were operated on in a hospital setting

NA

NA
NA

2,285,968

Both eyes: 1,005,826 (44\%)

One eye: 1,280,14221 (56\%)

$3,280,966^{\mathrm{e}}$

$11,873^{f}$

NA
(1): 1-50;
(2): $51-200$;
(3): 201-500;
(4): $501-1000$;
(5): $\geq 1001$

NA

NA

Patients with max. 2 cataract surgeries per beneficiary during the 2-year study timeframe; Medicare beneficiaries $\geq 65$ years

Records were excluded if data indicated the surgery was not performed, the procedure was a return to the operating room for a related procedure or due to data coding issues; surgeries performed in the last 42 days of 2004

NA

NA 
Table 3 Results from retrospective database analyses (thyroid and cataract surgery) (Continued)

\begin{tabular}{|c|c|c|c|}
\hline & Ayala and Yencha [15] (2015) & Chen et al. [16] (2014) & Keay et al. [17] (2012) \\
\hline & $\begin{array}{l}\text { (calcitriol) supplementation for pts. undergoing } \\
\text { total or completion thyroidectomy }\end{array}$ & & \\
\hline $\begin{array}{l}\text { Age, mean, yrs } \\
\text { (range) [SD] }\end{array}$ & $41.8(14-75) / 47.8(19-77)$ & $73(60-86)[3]$ & $N A\left(\geq 65^{h}\right)$ \\
\hline $\begin{array}{l}\text { Sex, female: } \\
\text { male, } n, \text { I vs C }\end{array}$ & $82: 27$ vs $25: 26$ & $13: 10$ & NA \\
\hline $\mathrm{BMI} \pm \mathrm{SD}$ & NA & NA & NA \\
\hline Risk Adjustment & NA & NA & $\begin{array}{l}\text { Age }(65-74,75-84, \geq 85 \\
\text { Gender, Race, Year, } \\
\text { Ambulatory surgery centre (No, Yes), } \\
\text { Surgeon experience in yrs. (1-10, } 11-20,21- \\
30, \geq 30)\end{array}$ \\
\hline $\begin{array}{l}\text { Other } \\
\text { influencing } \\
\text { factors } \\
\text { (Comorbidities } \\
\text { etc.), n (\%) }\end{array}$ & NA & $\begin{array}{l}\text { Shallow chamber: } 8 \text { (35); } \\
\text { Miosis: } 7 \text { (30); Restlessness: } 6 \\
\text { (26); } \\
\text { Floppy Iris: } 6 \text { (26); } \\
\text { Pseudoexfoliation: } 5 \text { (22); } \\
\text { Zonular dehiscence: } 5 \text { (22); } \\
\text { Small eye: } 1 \text { (4) }\end{array}$ & NA \\
\hline $\begin{array}{l}\text { Patients } \\
\text { excluded from } \\
\text { the analysis, } n \\
\text { (\%) }\end{array}$ & NA & NA & 165,452 and $35,068^{\mathrm{i}}$ \\
\hline \multicolumn{4}{|l|}{ Efficacy } \\
\hline Revision Surgery & NA & $23^{j}$ of 3339 & NA \\
\hline $\begin{array}{l}\text { Re-admission }{ }^{k}, \mathrm{n} \\
(\%), \text { I vs C }\end{array}$ & $O^{\prime}$ vs NA & NA & NA \\
\hline $\begin{array}{l}\text { Surgical volume, } \\
\text { n (cases/year) - } \\
\text { Risk of } A E \\
\text { related } \\
\text { to surgery }\end{array}$ & NA & $\begin{array}{l}411-3.75 \\
536-0.37 \\
1056-0.28 \\
1336-0.29 \\
\text { PCR in } 23(0.68) \text { in total }\end{array}$ & 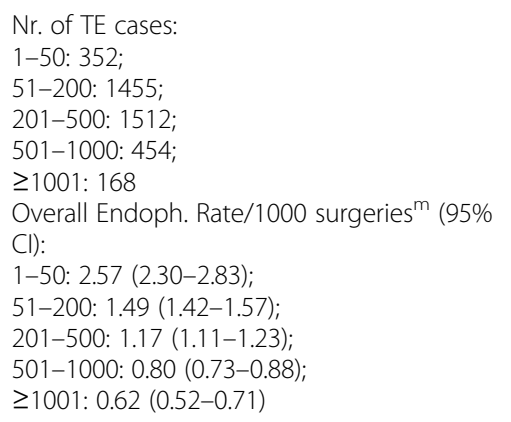 \\
\hline $\begin{array}{l}\text { Surgical volume, } \\
\text { n (cases/year) - } \\
\text { Risk of } A E \\
\text { related } \\
\text { to surgery } \\
\text { (continuation) }\end{array}$ & & & 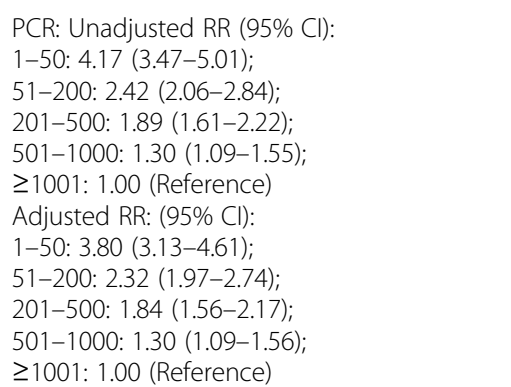 \\
\hline
\end{tabular}

\section{Safety}

SAE

AEs, Volume, $n$ (\%),
NA

19 pts. of 160 pts. $(11,90 \%)^{\text {n }}$

Transient hypercalcemia: 5\%;

Temporary vocal cord paralysis: $2.5 \%$;

Bilateral vocal cord paralysis: $0.63 \%$

Inadvertent transection of the RLN: $0.63 \%$;

Post-operative seromas requiring aspiration:
NA

NA
NA

NA 
Table 3 Results from retrospective database analyses (thyroid and cataract surgery) (Continued)

\begin{tabular}{lll}
\hline Ayala and Yencha [15] (2015) & Chen et al. [16] (2014) & Keay et al. [17] (2012) \\
\hline 1.9\%; \\
Post-operative hematoma requiring aspiration: \\
$1.25 \%$
\end{tabular}

NA Not available, $P C R$ Post Cataract Endophthalmitis, SDS Same day surgery, SDT Same-day thyroidectomy, TE Total Endophthalmitis, $T$ Thyroidectomy, RLN Recurrent laryngeal nerve, $R R$ Relative Risk

aPatient who were eligible for same day discharge were observed typically for 2-4 h. Patients with significant co-morbidities, lack of social support, and/or patients not comfortable with outpatient recovery were admitted for observation

${ }^{\mathrm{b}}$ Forty-three points were kept for $23 \mathrm{~h}$ observation and $17(40 \%)$ of these patients were found to have social factors requiring an overnight stay (due to long distance, absence of responsible adult caregiver); remaining 26 pts. requiring a $23 \mathrm{~h}$ observation had significant co-morbidities

'Own calculation on the basis of the given numbers of patients (absolute and relative) with surgery on both eyes.

'Outpatient (Intervention) vs Inpatient (Comparator)

e 35,068 surgeries could not have been attributed to a specific surgeon and also contain surgeries for which surgeon characteristics data were missing. Hence in the analysis $3,245,898$ were included.

fOwn calculation on the basis of the descriptive statistics of the endophthalmitis rate by annual Medicare surgical volume found in Table 4.

${ }^{9}$ Thresholds for MVS classification (i.e. low, medium, high) is not clear

${ }^{\mathrm{h}}$ Age info is not given in detail

${ }^{1} 165,452$ Surgeries performed in the last 42 days in 2004 were excluded and in the analysis of the endophthalmitis rate by annual medicare surgical volume 35,068 surgeries with unique physician identification numbers that cannot be attributed to a specific surgeon and surgeries for which surgeon characteristics data were missing

${ }^{\mathrm{j}}$ Five of the 23 patients did not have sufficient support to place a posterior chamber or sulcus intraocular cataract lenses (IOL) and required placement of anterior chamber IOLs

${ }^{\mathrm{k}}$ Re-admission includes admission for 23-h observation or full admission (observation longer than $24 \mathrm{~h}$ )

'One pt. was discharged from the ER for symptoms of paresthesias with normal calcium levels

${ }^{m}$ This rate is overall for all surgeries within a specific annual volume category and does not reflect the average rate of endophthalmitis within each category

${ }^{n}$ It was unclear what AE occurred in the respective intervention arm

Table 4 List of outcomes used in the assessment

\begin{tabular}{|c|c|c|c|c|c|c|c|}
\hline Intervention & $\begin{array}{l}\text { Thyroid surgery } \\
\text { (thyroidectomy) }\end{array}$ & $\begin{array}{l}\text { Cataract surgery } \\
\text { (phacoemulsification) }\end{array}$ & $\begin{array}{l}\text { Hip } \\
\text { arthroscopy }\end{array}$ & $\begin{array}{l}\text { Carpal tunnel } \\
\text { release } \\
\text { (decompression } \\
\text { surgery) }\end{array}$ & $\begin{array}{l}\text { Rotator cuff } \\
\text { repair }\end{array}$ & $\begin{array}{l}\mathrm{ACL} \\
\text { reconstruction }\end{array}$ & Meniscectomy \\
\hline $\begin{array}{l}\text { Intervention } \\
\text { description }\end{array}$ & $\begin{array}{l}\text { Removal of part } \\
\text { or all of the } \\
\text { thyroid gland }\end{array}$ & $\begin{array}{l}\text { Removal of the lens of } \\
\text { the eye and } \\
\text { replacement it with an } \\
\text { artificial lens }\end{array}$ & $\begin{array}{l}\text { Surgical } \\
\text { procedure used } \\
\text { for diagnosis as } \\
\text { well as } \\
\text { treatment of } \\
\text { hip related } \\
\text { disorders }\end{array}$ & $\begin{array}{l}\text { Surgical treatment } \\
\text { of the carpal } \\
\text { tunnel syndrome - } \\
\text { compressed nerves } \\
\text { in the wrist }\end{array}$ & $\begin{array}{l}\text { Surgical } \\
\text { treatment of } \\
\text { rotator cuff } \\
\text { disorders } \\
\text {-inflamed or } \\
\text { irritated } \\
\text { shoulder } \\
\text { tendons }\end{array}$ & $\begin{array}{l}\text { Surgical } \\
\text { treatment of } \mathrm{ACL} \\
\text { injuries -tears or } \\
\text { sprains }\end{array}$ & $\begin{array}{l}\text { Surgical } \\
\text { treatment of } \\
\text { meniscal } \\
\text { injuries }\end{array}$ \\
\hline $\begin{array}{l}\text { Volume } \\
\text { perspective }\end{array}$ & Surgeon & Surgeon & Surgeon & Surgeon & $\begin{array}{l}\text { Surgeon/ } \\
\text { Hospital }\end{array}$ & Surgeon/Hospital & $\begin{array}{l}\text { Surgeon/ } \\
\text { Hospital }\end{array}$ \\
\hline Outcomes & $\begin{array}{l}\text { - Admission for } \\
23 \mathrm{~h} \\
\text { observation } \\
\text { - Full admission } \\
\text { (longer than } \\
23 \mathrm{~h} \text { ) } \\
\text { - AEs }\end{array}$ & $\begin{array}{l}\text { - Risk of revision } \\
\text { surgery } \\
\text { - Risk-adjusted inci- } \\
\text { dence of post- } \\
\text { surgery } \\
\text { endophthalmitis }\end{array}$ & $\begin{array}{l}\text { - Risk of } \\
\text { reoperation } \\
\text { - Survival rates } \\
\text { at two, five, } \\
\text { and } 10 \text { yrs. }^{a} \\
\text { - AEs }\end{array}$ & $\begin{array}{l}\text { - BCTQ score }{ }^{\mathrm{b}} \text { was } \\
\text { measured at } 6 \\
\text { weeks, } 3 \text { months, } \\
\text { and } 6 \text { months full } \\
\text { admission (longer } \\
\text { than } 23 \text { h) } \\
\text { - Reduction in pain } \\
\text { score units on } \\
\text { VAS }{ }^{\mathrm{c}} \text { measured } 6 \\
\text { weeks, } 3 \text { months, } \\
\text { and } 6 \text { months } \\
\text { - AEs }\end{array}$ & $\begin{array}{l}\text { - Non-routine } \\
\text { disposition of } \\
\text { patients at } \\
\text { discharge }{ }^{d a} \\
\text { - LOS } \text { Loa }^{\text {ea }} \\
\text { - Mean } \\
\text { operating }_{\text {time }}^{f}\end{array}$ & $\begin{array}{l}\text { - Non-routine } \\
\text { disposition of } \\
\text { patients at } \\
\text { discharge } \\
\text { - Inpatient } \\
\text { hospital } \\
\text { admissions or } \\
\text { emergency } \\
\text { department } \\
\text { visits within } 30 \\
\text { days of } \\
\text { surgery } \\
\text { - Mean operating } \\
\text { time }\end{array}$ & $\begin{array}{l}\text { - Non-routine } \\
\text { disposition of } \\
\text { patients at } \\
\text { discharge }{ }^{a} \\
\text { - Mean } \\
\text { operating } \\
\text { time }\end{array}$ \\
\hline
\end{tabular}

Abbreviations: ACL Anterior cruciate ligament, AEs Adverse events, BCTQ Boston Carpal Tunnel Syndrome Questionnaire, hrs hours, LOS Length of stay, VAS Visual analogue scale, yrs. years

${ }^{a}$ refers to those outcomes that we considered crucial

${ }^{\mathrm{b}}$ Two domains of BCTQ were assessed in particular: the Symptom Severity Scale (11 items) and the Functional Status Scale (8 items). Each item consists of five response categories ranging from one to five, where higher score represents worse symptoms/lower level of function

'VAS ranged from 0 to 100

${ }^{\mathrm{d}}$ Non-routine disposition includes transfer to another hospital, skilled nursing facility, intermediate care facility, or home health care (health care provided at home by licensed health professionals)

eLOS was divided into two categories: less than 1 day and greater than or equal to 1 day - that was termed extended length of stay

foperating room time was calculated in minutes for every procedure and defined as the total time spent in the operating room exclusive of preoperative and postoperative time

${ }^{\mathrm{g}}$ Assessed from hospital perspective only 


\section{Study characteristics}

While two studies were single center analyses [21, 25], six studies were analyses of health care databases [2224, 26-28]. Seven studies were conducted in the US $[21-26,28]$ and the eighth study was conducted in the Netherlands [27]. Information about study sponsors was not disclosed in five studies [21-23, 25, 26], two studies were funded by the National Institutes of Health/National Institute for Arthritis and Musculoskeletal and Skin Diseases $[27,28]$, and one study was funded by the National Eye Institute [24]. Two studies did not report on conflict of interests (COI) [23, 26], four studies reported that none of the authors had COI [22, 24, 25, 27], and two studies reported COI of one of their authors [21, 28]. The dates of data collection in all the studies were between 1997 and 2015.

All the studies gathered data on the outpatient setting and while four studies analyzed the VOR from the perspective of surgeons $[24,25,27,28]$, one analyzed it from the hospital perspective [22], and three from the perspectives of both surgeons as well as hospitals [21, $23,26]$. Follow-up time was not reported in five studies [21, 23-26], it was 10 years in [28], 6 months in [27], and 30 days in [22].

\section{Patient characteristics}

The analysis of primary hip arthroscopy included 7836 patients and 8267 procedures that were performed by 295 surgeons in 137 centers [28]. The analysis of carpal tunnel release included 1345 patients/procedures (712 patients not followed-up) performed by 17 surgeons in 11 centers [27]. The analysis of rotator cuff repair included 9973 patients (961 not followed-up) [26], ACL reconstruction included 45,262 patients (14,050 not followed-up) [22, 23], and the analysis of meniscectomy included 123,012 patients (72,585 not followed-up) [23] . The number of procedures, surgeons, or surgical centers was not reported in the three studies above. For the single center analysis of thyroidectomy, 109 outpatient and 51 inpatient patients were included with 35 and 26 procedures respectively [21]. For the analysis of cataract surgery, 2,289,307 patients were included (200,520 not followed-up) with $3,280,966$ procedures conducted by 22,877 surgeons in an unclear number of centers (except for four surgeons that were part of a single center analysis [25]). Because of the retrospective nature of the studies, loss to follow-up was not reported.

Surgeon as well as hospital volume was categorized into low, medium, and high (very high in one study [28]) and the thresholds differed with interventions. The low volume threshold ranged from six to 411 interventions, while the high (or very high) threshold ranged from 30 to 1336 interventions per year. Inclusion and exclusion criteria were heterogeneous as they varied with interventions. Co-interventions were reported in four studies $[21,22,26,27]$ and the mean age ranged from 29,4 to 73 years. Study characteristics and results of included studies are displayed in Tables 2 and 3.

\section{Clinical effectiveness}

Concerning cataract surgery, inverse VOR was observed [24]. The surgeon volume rates ranged from 1 to 50 (1), 51-200 (2), 201-500 (3), 501-1000 (4) and $\geq 1001$ (5). The number of cases per surgeon was inversely correlated with the adverse event of posterior capsule rupture (PCR), where PCR and vitreous loss (VL) rate were $3.75 \%$ for low volume and $0.29 \%$ for very high volume surgeons [25]. The relative risk (RR) for endophtalmitis was 4-fold between low and very high volume surgeons [24]. After adjusting for risk factors the RR in category (1) was still 3.8-fold higher than in the reference category (5).

Concerning thyroidectomy, there was no VOR observed, but it was suggested that thyroidectomy is safe also in low volume centers as in the single low volume center, no cases of readmission occurred [21]. The surgeon volume amounted to 10 thyroid surgical cases per year on average, while the hospital volume averaged of 20 thyroid surgical cases per year.

Concerning rotator cuff repair, VOR was observed. The surgeon volume rates ranged from low $(<15)$, medium (15-30), to high $(\geq 30)$, while hospital volume rates ranged from low $(<75)$, medium $(75-200)$, to high $(\geq 200)$. Patients of low volume surgeons were 2.8 time more likely to have nonroutine disposition at discharge, while low volume hospitals were 2.1 times more likely to discharge patients with nonroutine dispositions. Surgeon-related mean operating time was 10 min shorter and hospital-related mean operating time was $6 \mathrm{~min}$ shorter for high volume compared to low volume surgeons/hospitals. Length of stay (LOS) was 2.3 times longer for low volume surgeons and 0.5 times for low volume hospitals compared to high volume surgeons/ hospitals [26].

Concerning hip arthroscopy, VOR was observed. Surgeon yearly volume rates ranged from low $(<102)$, medium (102-164), high (164-340), to very high $(\geq 340)$. The survival rates for very high volume surgeons were 11.1-24.9\% higher than for low volume and the hazard ratio for reoperation (with reference value of low volume) was 0.17 for very high volume surgeons [28].

Concerning open carpal tunnel release, VOR was not observed. Surgeon yearly volume rates ranged from low (6-44), medium (47-71), to high (75-163). BCTQ score did not vary with volume at all while the difference on the VAS scale was 1 point (out of 100) between low and high volume surgeons (18 vs. 19 points) [27]. Such 
difference is below the threshold of the minimal clinically important difference [15].

Concerning ACL reconstruction, VOR was observed. The surgeon volume rates ranged from low $(<25)$, medium (25-75), to high ( $\geq 75)$ and hospital volume rates ranged from low $(<125)$, medium (125-300), to high $(\geq 300)$. The odds ratio for hospital based acute care within 30 days (with reference of low volume hospitals) was 0.47 for high volume hospitals [22]. Low volume surgeons were 4.5 times more likely and low volume hospital 3.33 times more likely to have nonroutinely discharged patients compared to high volume surgeons [23]. Furthermore, low volume surgeons had a $27 \mathrm{~min}$ longer and low volume hospitals 21 min longer mean operating time than high volume surgeons/ hospitals [23].

Concerning meniscectomy, VOR was observed. The surgeon volume rates ranged from low $(<75)$, medium (75-175), to high $(\geq 175)$ and the hospital volume rates ranged from low $(<600)$, medium (600-1200), to high $(\geq 1200)$. The low volume surgeons were 2.8 times and low volume hospitals were 8 times more likely to have nonroutinely disposed patients at discharge than high volume surgeons/hospitals [23]. In terms of mean operating time, both low volume surgeons and low volume hospitals had a longer mean operating time by $19 \mathrm{~min}$ compared to high volume surgeons/hospitals [23].

\section{Safety}

The only safety related data reported were without its relationship to surgeon/hospital volume. In the hip arthroscopy study, $0.2 \%$ of patients experienced procedural complication at 30 days post intervention - the complications were: myocardial infarction ileus, pneumonia, sepsis, mechanical complication, hardware failure, deep vein thrombosis/pulmonary embolism, wound infection, dislocation/iatrogenic instability, major bleed [28]. In the carpal tunnel release study, $1.6 \%$ of patients experienced procedural complications such as wound infections, wound dehiscence [27].

In the thyroidectomy study [21], 19 of the 160 patients experienced complications. Complications included transient hypercalcemia (5\%), temporary vocal cord paralysis (2.5\%), post-operative seromas requiring aspiration (1.9\%), post-operative hematoma requiring aspiration (1.25\%), bilateral vocal cord paralysis $(0.63 \%)$, and inadvertent transection of the recurrent laryngeal nerve (0.63\%).

\section{Discussion}

\section{Clinical effectiveness and safety}

To our knowledge, this is the first systematic review on minimum volume standards (MVSs) applied to the general setting of day surgery. The 2012 report of the German Institute for Quality and Efficiency in Healthcare
(IQWiG) on effects of minimum volume regulations was set out to evaluate outpatient evidence only, however, it also included inpatient data [16].

\section{Summary of evidence from retrospective database analysis}

Focusing exclusively on the day surgery setting, we found no prospective or controlled trials for the analyses of clinical effectiveness and safety. We found eight retrospective database analyses on seven different indications, but none reported on the volume-outcome relationship (VOR) with respect to safety. Each indication included the following number of patients:

$\beta$ thyroidectomy - 109 outpatient (and 51 inpatient) patients [21],

$\beta$ cataract surgery $-2,289,307$ patients $[24,25]$

$\beta$ primary hip arthroscopy -7836 patients [28],

$\beta$ open carpal tunnel release - 1345 patients [27],

$\beta$ rotator cuff repair - 9973 patients [26]

$\beta$ ACL reconstruction - 45,262 patients $[22,23]$

$\beta$ and meniscectomy $-123,012$ patients [23].

All interventions (except for carpal tunnel release and thyroidectomy) confirmed the VOR, however, none established MVS for the respective interventions.

\section{Gaps in evidence}

While the VOR has some standing in the inpatient setting [14], it is argued that that relationship is based on low quality of evidence [17]. That is even more true for the day surgery setting and because of the fact that day surgery centers may operate independently from hospitals and so miss on the safety net in the form of emergency departments, quality assurance in day surgery is of even more importance. That places the extra emphasis on day surgery interventions to go well in the first place and hence also on quality assurance measures such as MVSs.

\section{Relevance of evidence}

Due to these gaps in evidence, the relevance of the current evidence base to relative effectiveness assessment of MVSs is questionable. Retrospective database analyses do not fulfil the evidence-based medicine standards as they are prone to a spectrum of biases. For that reason, their conclusions are applicable only in part. In their favor plays the relatively robust body of evidence from the inpatient setting, relatively high number of patients included in the day surgery studies, and studies supporting no significant difference in outcomes between the settings [2]. Against it plays the poor internal and external validity of the present evidence base and 
the critical considerations related to MVSs in general (outlined below).

\section{Internal and external validity of the present evidence base}

Concerning effectiveness and safety of MVSs as assessed by the ISPOR checklist in Table 5 , the quality of the evidence base is very low. The main reasons are the retrospective design of all the studies [21-28], the lack of justification for its use [21, 23, 25-27], or the lack of a priori data analysis plan [21-28]. Further reasons include unclear eligibility criteria [21, 22, 25, 27, 28], lack of justification for the statistical models used [21-23, 25-28], lack of interpretation of the statistical findings in terms of their clinical or economical evidence [22, 23, 25-28], and limited recognition of the generalizability of the retrospective study design [21, 23, 25-27].

Moreover, the validity of the endpoints used is also questionable. None of the safety endpoints were reported in relation to surgeon or hospital volumes, only one study reported on a mortality endpoint [28], three on morbidity endpoints $[22,23,26]$, one on disease specific QoL endpoint [27], and none of patient satisfaction. Day surgery related outcomes such as frequency of hospital infections or venous thrombosis embolisms were not reported in any of the studies.

External validity of the data is questionable as well. Even though the conclusions of all the included studies are based on clinical practice data, the potential patient selection bias or the retrospective study design and the questionable generalizability undermine the external validity. Generalizability of the data is put in question because all the studies (except for [27]) were conducted in the USA where the definition of day surgery and outpatient surgery may vary [5].

\section{Critical considerations and contradictory evidence}

A critical synthesis has to be made to draw attention on the complexity of the VOR and thereof derived administrative MVSs. It is crucial to consider the overdeterministic nature of this relationship in order to avoid possible methodological drawbacks in the study design and to guarantee explanatory power $[12,13]$.

Without a doubt, taking into account risk-adjustment and case mix is imperative as the first step toward getting unbiased estimates of the effect of volume on outcome, but volume of surgeries can only be a proxy for higher quality. Halm et al. point out that besides general methodical shortcomings, studies investigating the VOR were not able to determine what surgical abilities or management skills of the surgery unit are enhanced by volume and why they should be uniquely related to volume [11].
Whether high-quality hospitals or surgeons are more likely to be sought by patients in the first place and are therefore capable of accumulating a higher-volume is also important to consider. Word of mouth takes a substantial part in the decision of where to undergo treatment that is often neglected in studies and health care research [10]. Also, the question whether patients of high-volume providers are more likely to be selected into treatment by the provider compared to low-volume surgeons or hospitals is mainly unanswered [11].

When conducting volume-outcome analysis, one has to be cautious not to fall in a mono-causal or reverse causality trap when establishing links between two or more variables. An observed correlation does not necessarily indicate causation. Against this backdrop, it is important to synthesize the various approaches to emphasize the complexity of the VOR and its derived policies. A scientific and surgery specific examination of the study situation is necessary to establish evidencebased minimum quantities. Quality is influenced by other factors such as the application of the best treatment methods. These factors should always supplement quality assurance focused on minimum quantities.

\section{Limitations}

The evidence base found was only partly relevant for answering the research questions. The retrospective study design can at best show correlations between surgeon/ hospital volumes and day surgery outcomes, however, its results are of limited certainty and none of the studies answered the question on the threshold MVSs.

In the systematic literature search, we only found VOR data on eight interventions, which, however, is not a representative sample of all the interventions eligible for day surgery.

Also, the consistency of definitions of the included studies is questionable. The reason is that the US' use of the term day surgery may include $23 \mathrm{~h}$ stay surgery, which in the EU is seen as an inpatient surgery with a 1 day LOS.

Furthermore, the following studies on arthroscopy, meniscal repair, and colonoscopy [29-31] were excluded from the analysis even though they met the inclusion criteria. The reason was that they were found in an additional search in the end stage of the report when including them was no longer feasible. Additional reason for not including them was their assumed low marginal utility as quality of all studies was low due to their retrospectives study design.

\section{Conclusion}

The need for a shift of surgical interventions from the inpatient setting to the day surgery setting is advocated in the international literature. The reasons for the shift 
Table 5 ISPOR task force checklist for quality assessment of retrospective database studies [29]

\begin{tabular}{|c|c|c|c|c|c|c|c|c|}
\hline Study reference/ID & $\begin{array}{l}\text { Degan } \\
\text { et al. [10] } \\
(2017)\end{array}$ & $\begin{array}{l}\text { Evers } \\
\text { et al. } \\
{[11]} \\
(2018)\end{array}$ & $\begin{array}{l}\text { Jain } \\
\text { et al. } \\
{[12]} \\
(2005)\end{array}$ & $\begin{array}{l}\text { Jain } \\
\text { et al. } \\
{[13]} \\
(2004)\end{array}$ & $\begin{array}{l}\text { Liu } \\
\text { et al. } \\
{[14]} \\
(2018)\end{array}$ & $\begin{array}{l}\text { Ayala and } \\
\text { Yencha [15] } \\
(2015)\end{array}$ & $\begin{array}{l}\text { Chen } \\
\text { et al. } \\
{[16]} \\
(2014)\end{array}$ & $\begin{array}{l}\text { Keay } \\
\text { et al. } \\
{[17]} \\
(2012)\end{array}$ \\
\hline $\begin{array}{l}\text { 1. Relevance: Have the data attributes been described in } \\
\text { sufficient detail for decision makers to determine whether } \\
\text { there was a good rationale for using the data source, the } \\
\text { data source's overall generalizability, and how the findings } \\
\text { can be interpreted in the context of their own organization? }\end{array}$ & Yes & Yes & Yes & Yes & Yes & Yes & Yes & Yes \\
\hline $\begin{array}{l}\text { 2. Reliability and Validity: Have the reliability and validity of } \\
\text { the data been described, including any data quality checks } \\
\text { and data cleaning procedures? }\end{array}$ & No & Yes & Yes & Yes & No & No & Yes & Yes \\
\hline $\begin{array}{l}\text { 3. Linkages: Have the necessary linkages among data sources } \\
\text { and/or different care sites been carried out appropriately, } \\
\text { taking into account differences in coding and reporting } \\
\text { across sources? }\end{array}$ & Yes & NA & Yes & Yes & Yes & $N A^{a}$ & $N A^{a}$ & Yes \\
\hline $\begin{array}{l}\text { 4. Eligibility: Have the authors described the type of data } \\
\text { used to determine member eligibility? }\end{array}$ & Yes & Yes & Yes & Yes & Yes & No & No & Yes \\
\hline $\begin{array}{l}\text { 5. Data analysis plan: was a data analysis plan, including } \\
\text { study hypotheses, developed a priori? Was the study } \\
\text { conducted prospectively? }\end{array}$ & Partial $^{b}$ & Partial $^{b}$ & Partial $^{b}$ & Partial $^{b}$ & Partial $^{b}$ & $\mathrm{No}^{\mathrm{c}}$ & No & Partial $^{b}$ \\
\hline $\begin{array}{l}\text { 6. Design selection: has the investigator provided a rationale } \\
\text { for the particular research design? }\end{array}$ & No & No & No & No & No & No & No & Partial $^{d}$ \\
\hline $\begin{array}{l}\text { 7. Research design limitations: did the author identify and } \\
\text { address potential limitations of that design? }\end{array}$ & Yes & No & No & No & Yes & No & No & Partial \\
\hline $\begin{array}{l}\text { 8. Treatment effect: for studies that are trying to make } \\
\text { inferences about the effects of an intervention, does the } \\
\text { study include a comparison group and have the authors } \\
\text { described the process for identifying the comparison group } \\
\text { and the characteristics of the comparison group as they } \\
\text { relate to the intervention group? }\end{array}$ & NA & NA & NA & NA & NA & NA & NA & NA \\
\hline $\begin{array}{l}\text { 9. Sample selection: have the inclusion and exclusion criteria } \\
\text { and the steps used to derive the final sample from the initial } \\
\text { population been described? }\end{array}$ & Partial $^{e}$ & Partial $^{f}$ & Yes & Yes & Partial $^{9}$ & No & Partial $^{f}$ & Yes \\
\hline $\begin{array}{l}\text { 10. Eligibility: are subjects eligible for the time period over } \\
\text { which measurement is occurring? }\end{array}$ & NA & NA & NA & NA & NA & NA & NA & NA \\
\hline $\begin{array}{l}\text { 11. Censoring: were inclusion/exclusion or eligibility criteria } \\
\text { used to address censoring and was the impact on study } \\
\text { findings discussed? }\end{array}$ & Partial $^{h}$ & Partial $^{\text {h }}$ & Partial $^{\text {h }}$ & Partial $^{\text {h }}$ & Partial $^{h}$ & No & Partial $^{h}$ & Yes \\
\hline $\begin{array}{l}\text { 12. Operational definitions: are case (subjects) and end point } \\
\text { (outcomes) criteria explicitly defined using diagnosis, drug } \\
\text { markers, procedure codes, and/or other criteria? }\end{array}$ & Yes & Yes & Yes & Yes & Yes & Partial $^{\mathrm{i}}$ & Yes & Yes \\
\hline $\begin{array}{l}\text { 13. Definition validity: have the authors provided a rationale } \\
\text { and/or supporting literature for the definitions and criteria } \\
\text { used and were sensitivity analyses performed for definitions } \\
\text { or criteria that are controversial, uncertain, or novel? }\end{array}$ & NA & NA & NA & NA & NA & NA & NA & NA \\
\hline $\begin{array}{l}\text { 14. Timing of outcome: is there a clear temporal (sequential) } \\
\text { relationship between the exposure and outcome? }\end{array}$ & NA & NA & NA & NA & NA & NA & NA & NA \\
\hline $\begin{array}{l}\text { 15. Event capture: are the data, as collected, able to identify } \\
\text { the intervention and outcomes if they actually occurred? }\end{array}$ & Yes & Yes & Yes & Yes & Yes & Yes & Yes & Yes \\
\hline $\begin{array}{l}\text { 16. Disease history: is there a link between the natural history } \\
\text { of the disease being studied and the time period for } \\
\text { analysis? }\end{array}$ & NA & NA & NA & NA & NA & NA & NA & NA \\
\hline $\begin{array}{l}\text { 17. Resource valuation: for studies that examine costs, have } \\
\text { the authors defined and measured an exhaustive list of } \\
\text { resources affected by the intervention given the perspective } \\
\text { of the study and have resource prices been adjusted to yield } \\
\text { a consistent valuation that reflects the opportunity cost of } \\
\text { the resource? }\end{array}$ & NA & NA & NA & NA & NA & NA & NA & NA \\
\hline 18. Control variables: if the goal of the study is to examine & Yes & Yes & Yes & Yes & Yes & Nonej & None $^{k}$ & Yes \\
\hline
\end{tabular}


Table 5 ISPOR task force checklist for quality assessment of retrospective database studies [29] (Continued)

\begin{tabular}{|c|c|c|c|c|c|c|c|c|}
\hline Study reference/ID & $\begin{array}{l}\text { Degan } \\
\text { et al. [10] } \\
(2017)\end{array}$ & $\begin{array}{l}\text { Evers } \\
\text { et al. } \\
{[11]} \\
(2018)\end{array}$ & $\begin{array}{l}\text { Jain } \\
\text { et al. } \\
{[12]} \\
(2005)\end{array}$ & $\begin{array}{l}\text { Jain } \\
\text { et al. } \\
{[13]} \\
(2004)\end{array}$ & $\begin{array}{l}\text { Liu } \\
\text { et al. } \\
{[14]} \\
(2018)\end{array}$ & $\begin{array}{l}\text { Ayala and } \\
\text { Yencha [15] } \\
(2015)\end{array}$ & $\begin{array}{l}\text { Chen } \\
\text { et al. } \\
{[16]} \\
(2014)\end{array}$ & $\begin{array}{l}\text { Keay } \\
\text { et al. } \\
{[17]} \\
(2012)\end{array}$ \\
\hline \multicolumn{9}{|l|}{$\begin{array}{l}\text { treatment effects, what methods have been used to control } \\
\text { for other variables that may affect the outcome of interest? }\end{array}$} \\
\hline $\begin{array}{l}\text { 19. Statistical model: have the authors explained the } \\
\text { rationale for the model/statistical method used? }\end{array}$ & No & No & No & No & No & Partial $^{\prime}$ & No & Yes \\
\hline $\begin{array}{l}\text { 20. Influential cases: have the authors examined the } \\
\text { sensitivity of the results to influential cases? }\end{array}$ & NA & NA & NA & NA & NA & NA & NA & NA \\
\hline $\begin{array}{l}\text { 21. Relevant variables: have the authors identified all } \\
\text { variables hypothesized to influence the outcome of interest } \\
\text { and included all available variables in their model? }\end{array}$ & Yes & Yes & Yes & Yes & Yes & $\mathrm{No}^{\mathrm{m}}$ & No & No \\
\hline $\begin{array}{l}\text { 22. Testing statistical assumptions: do the authors investigate } \\
\text { the validity of the statistical assumptions underlying their } \\
\text { analysis? }\end{array}$ & Yes & Yes & Yes & Yes & Yes & No & No & No \\
\hline $\begin{array}{l}\text { 23. Multiple tests: if analyses of multiple groups are carried } \\
\text { out, are the statistical tests adjusted to reflect this? }\end{array}$ & NA & NA & NA & NA & NA & NA & NA & NA \\
\hline $\begin{array}{l}\text { 24. Model prediction: if the authors utilize multivariate } \\
\text { statistical techniques in their analysis, do they discuss how } \\
\text { well the model predicts what it is intended to predict? }\end{array}$ & No & No & No & No & No & NA & NA & Yes \\
\hline $\begin{array}{l}\text { 25. Theoretical biases: have the authors provided a theory for } \\
\text { the findings and have they ruled out other plausible } \\
\text { alternative explanations for the findings? }\end{array}$ & Partial $^{n}$ & Partial $^{n}$ & Partial $^{n}$ & Partial $^{\mathrm{n}}$ & Partial $^{n}$ & Partial $^{n}$ & No & Yes \\
\hline $\begin{array}{l}\text { 26. Practical versus statistical significance: have the statistical } \\
\text { findings been interpreted in terms of their clinical or } \\
\text { economic relevance? }\end{array}$ & No & No & No & No & No & Yes & Partial $^{\circ}$ & Yes \\
\hline $\begin{array}{l}\text { 27. Generalizability: have the authors discussed the } \\
\text { populations and settings to which the results can be } \\
\text { generalized? }\end{array}$ & Partial $^{p}$ & No & No & No & Partial $^{p}$ & No & No & Yes \\
\hline Overall level of quality & Very low & Very low & $\begin{array}{l}\text { Very } \\
\text { low }\end{array}$ & $\begin{array}{l}\text { Very } \\
\text { low }\end{array}$ & $\begin{array}{l}\text { Very } \\
\text { low }\end{array}$ & Very low & Very low & Very low \\
\hline
\end{tabular}

${ }^{\text {a }}$ Single data source

${ }^{b}$ It is indicated in the text that a hypothesis was created a priori, but no more information is revealed

${ }^{c}$ It is not explicitly stated in the text that a hypothesis was created a priori

${ }^{d}$ They state that population-based studies can be generalized to the community more easily than center-specific studies because they represent the broad range of conditions under which surgeries are conducted on diverse populations in a wide range of settings by many surgeons with varying levels of experience

eOnly inclusion criteria were described

${ }^{\mathrm{f}}$ The inclusion/exclusion criteria were described only in part

${ }^{g}$ Only exclusion criteria were described

${ }^{\text {h}}$ Criteria were mentioned, but the impact on findings was not discussed

iNo explicit procedure codes were used

jOnly bivariate statistical analyses were conducted - no multivariate regression model was applied

${ }^{k}$ Only causative factors are listed descriptively

'Reasons for using the conducted statistical tests were given

monly a low volume hospital with outpatient thyroidectomy was the object of analysis

${ }^{n}$ The authors did not rule out other possible interpretations

${ }^{\circ}$ Authors refer only to one paper that also tests the respective hypothesis

${ }^{\mathrm{P}}$ It is stated that the current results are of limited generalizability

include organizational, ethical, economic, and medical aspects and quality assurance in the process is argued to be key. Because the VOR does have some standing in the inpatient setting, the role of MVSs applied to the day surgery setting was scrutinized in this report. Surely, identifying possible analogies between the inpatient and the day surgery settings can serve as a valuable decision-making foundation, however, the lack of evidence of clearly established MVSs, methodical issues, and the different nature of inpatient and day surgery settings make the simple transition of inpatient results to the day surgery setting questionable.

Not only is the theory of MVSs in part questionable, but also the results from our systematic review cannot offer any clear-cut MVS thresholds. This present report, however, provides some evidence in favor of VOR, even though it based on low quality retrospective dataanalyses. Two out of eight studies did not even suggest a VOR at all and so we argue that the application of MVSs should be done with caution. Moreover, because establishing the VOR and henceforth the MVSs is possible, 
quality prospective controlled evidence for the day surgery setting is required. Also, other quality assurance standards such as standards focusing on process and outcome quality should be taken into account.

In terms of adequate policy implications, if optimal surgery specific MVSs can be established as a quality standard and it is secured by a high-quality body of evidence for the VOR, then it is also indispensable to derive an appropriate public policy to disseminate this information to payers, health care consumers, and clinicians. Prima facie, there are three different policy approaches: (1.) utilizing the data on volumes and outcomes to enhance performance, (2.) adopting measures to limit the and (3.) publication or dissemination of the data on volume.

\section{Supplementary information}

Supplementary information accompanies this paper at https://doi.org/10. 1186/s12913-020-05724-2.

\section{Additional file 1.}

\section{Abbreviations}

AA: Association of Anesthetists; ACL: Anterior cruciate ligament; BADS: British Association for Day Surgery; COl: Conflict of interests; EUnetHTA: European Network for Health Technology Assessment; IAAS: International Association for Ambulatory Surgery; IQWiG: Institute for Quality and Efficiency in Healthcare; ISPOR: International Society for Pharmacoeconomics and Outcomes Research; LOS: Length of stay; GRADE: Grading of Recommendations Assessment, Development and Evaluation; MVS: Minimum volume standards; PCR: Posterior capsule rupture; REA: Rapid Relative Effectiveness Assessment; RR: Relative risk; VOR: Volume-outcome relationship

\section{Acknowledgements}

We are most thankful to Tarquin Mittermayr for his excellent help with the literature search.

\section{Authors' contributions}

MS drafted the outline of the study, initiated the literature search, and made the first draft of the manuscript. CS revised the outline of the study, reviewed the included literature, and contributed to every revision of the manuscript. Both authors approve of the submitted manuscript.

\section{Author's information}

Not applicable.

\section{Funding}

This research was funded by the Ludwig Boltzmann Institute for Health Technology Assessment, Vienna, Austria. Both authors were employees of the institute at the time of writing of the study. The funder had no role in study design, data collection and analysis, decision to publish, or preparation of the manuscript. The Ludwig Boltzmann Institute for Health Technology Assessment changed in Austrian Institute for Health technology Assessment in March 2020

\section{Availability of data and materials}

Data generated or analyzed during this study are included in this published report: Stanak, M. and Strohmaier, C. (2019): Minimum volume standards for quality assurance in day surgery: Fundamentals and Systematic Review. HTAProjektbericht 125.

Ethics approval and consent to participate Not applicable.
Consent for publication

Not applicable.

\section{Competing interests}

Both authors, MS and CS, declare no conflict of interests.

Received: 15 June 2020 Accepted: 8 September 2020

Published online: 18 September 2020

\section{References}

1. International Association for Ambulatory Surgery. Ambulatory Surgery with Extended Recovery. 2019; Available from: https://www.iaas-med.com/index. php/iaas-recommendations/extended-recovery-facilities..

2. Castoro C, Bertinato L, Baccaglini U, Drace C, McKee M, et al. Day surgery : making it happen. WHO Regional Office for Europe: Copenhagen; 2007.

3. British Association of Day Surgery. BADS Directory of Procedures. London: British Association of Day Surgery; 2019.

4. Philip BK. Day care surgery: the United States model of health care. Ambul Surg. 2012;17:81-2..

5. Quemby DJ, Stocker ME. Day surgery development and practice: key factors for a successful pathway. BJA Educ. 2013;14(6):256-61.

6. Fischer S, Zechmeister-Koss I. Tageschirugie. Systematischer review HTAProjektbericht nr. 64. Ludwig Boltzmann Institut für Health Technology Assessment: Wien; 2012.

7. Alderwich H, Robertson R, Appleby J, Dunn P, Maguire D. Better value in the NHS - the role of changes in clinical practice. London: The King's Fund; 2015.

8. Bailey CR, Ahuja M, Bartholomew K, Bew S, Forbes L, Lipp A, et al. Guidelines for day-case surgery 2019. Anaesthesia. 2019;74(6):778-92..

9. Morche J, Renner D, Pietsch B, Kaiser L, Brönneke J, Gruber S, et al. International comparison of minimum volume standards for hospitals. Health Policy. 2018;122(11):1165-76..

10. Martin S. Word-of-mouth in the health care sector: a literature analysis of the current state of research and future perspectives. Int Rev Public Nonprofit Marketing. 2017;14(1):35-56..

11. Halm EA, Lee C, Chassin MR. How is volume related to quality in health care? A systematic review of the research literature. In: lo $\mathrm{M}$, editor. Interpreting the volume-outcome relationship in the context of health care quality. Workshop Summary Washington, DC: The National Academies Press; 2000.

12. Luft HS, Hunt SS, Maerki SC. The volume-outcome relationship: practicemakes-perfect or selective-referral patterns? Health Serv Res. 1987;22(2):15782.

13. Halm EA, Lee C, Chassin MR. Is volume related to outcome in health care? A systematic review and Methodologic critique of the literature. Ann Intern Med. 2002;137(6):511-20..

14. Morche J, Mathes T, Pieper D. Relationship between surgeon volume and outcomes: a systematic review of systematic reviews. Systematic Rev. 2016; 5(1):204..

15. Olsen MF, Bjerre E, Hansen MD, Hilden J, Landler NE, Tendal B, et al. Pain relief that matters to patients: systematic review of empirical studies assessing the minimum clinically important difference in acute pain. BMC Med. 2017;15(1):35.

16. Institute for Quality and Efficiency in Health Care. Literature search and evidence assessment to examine the effects of minimum volume regulations according to the G-BA directive on outpatient treatment in hospitals. Cologe: IQwiG; 2012. Contract No.: V11-01.

17. Geraedts $M$, de Cruppe W. Controversial study results in relation to minimum volume standards. Z Arztl Fortbild Qualitatssich. 2006;100(2):87-91 Kontroverse Studienergebnisse zur Mindestmengenproblematik..

18. Motheral B, Brooks J, Clark MA, Crown WH, Davey P, Hutchins D, et al. A checklist for retrospective database studies - report of the ISPOR task force on retrospective databases. Value Health. 2003:6(2):90-7..

19. European network for health technology assessment (EUnetHTA). Endpoints used in relative effectiveness assessment: surrogate endpoints. 2015. 06/06/ 2016. Available from: http://www.eunethta.eu/outputs/endpoints-usedrelative-effectiveness-assessment-surrogate-endpoints-amended-ja1guideline-f..

20. European network for health technology assessment (EUnetHTA). EUnetHTA Guidelines: EUnetHTA; 2015. Available from: http://www.eunethta.eu/ eunethta-guidelines. Cited 06/06/2016.. 
21. Ayala MA, Yencha MW. Outpatient thyroid surgery in a low-surgical volume hospital. World J Surg. 2015;39(9):2253-8..

22. Liu J, Kim DH, Maalouf DB, Beathe JC, Allen AA, Memtsoudis SG. Thirty-day acute health care resource utilization following outpatient anterior cruciate ligament surgery. Reg Anesth Pain Med. 2018;43(8):849-53..

23. Jain N, Pietrobon R, Guller U, Shankar A, Ahluwalia AS, Higgins LD. Effect of provider volume on resource utilization for surgical procedures of the knee. Knee Surg Sports Traumatol Arthrosc. 2005;13(4):302-12..

24. Keay L, Gower EW, Cassard SD, Tielsch JM, Schein OD. Postcataract surgery endophthalmitis in the United States: analysis of the complete 2003 to 2004 Medicare database of cataract surgeries. Ophthalmology. 2012;119(5):91422.

25. Chen M, Lamattina KC, Patrianakos T, Dwarakanathan S. Complication rate of posterior capsule rupture with vitreous loss during phacoemulsification at a Hawaiian cataract surgical center: a clinical audit. Clin. 2014:8:375-8.

26. Jain NB, Pietrobon R, Guller U, Ahluwalia AS, Higgins LD. Influence of provider volume on length of stay, operating room time, and discharge status for rotator cuff repair. J Shoulder Elb Surg. 2005;14(4):407-13..

27. Evers S, Jansen MC, Slijper HP, de Haas N, Smit X, Porsius JT, et al. Hand surgeons performing more open carpal tunnel releases Do not show better patient outcomes. Plast Reconstr Surg. 2018;141(6):1439-46..

28. Degen RM, Pan TJ, Chang B, Mehta N, Chamberlin PD, Ranawat AS, et al. Risk of failure of primary hip arthroscopy-a population-based study. J Hip Preserv Surg. 2017:4(3):214-23.

29. Lyman S, Hidaka C, Valdez AS, Hetsroni I, Pan TJ, Do H, et al. Risk factors for Meniscectomy after meniscal repair. Am J Sports Med. 2013:41(12):2772-8.

30. Wang P, Xu T, Ngamruengphong S, Makary MA, Kalloo A, Hutfless S. Rates of infection after colonoscopy and osophagogastroduodenoscopy in ambulatory surgery centres in the USA. Gut. 2018;67(9):1626..

31. Chukmaitov A, Devers K, Harless DW, Menachemi N, Brooks RG. Strategy, structure, and patient quality outcomes in ambulatory surgery centers (1997-2004). Med Care Res Rev. 2010;68(2):202-25.

\section{Publisher's Note}

Springer Nature remains neutral with regard to jurisdictional claims in published maps and institutional affiliations.

Ready to submit your research? Choose BMC and benefit from:

- fast, convenient online submission

- thorough peer review by experienced researchers in your field

- rapid publication on acceptance

- support for research data, including large and complex data types

- gold Open Access which fosters wider collaboration and increased citations

- maximum visibility for your research: over $100 \mathrm{M}$ website views per year

At $\mathrm{BMC}$, research is always in progress.

Learn more biomedcentral.com/submissions 